\title{
ACTIVE-TWIST ROTOR CONTROL APPLICATIONS FOR UAVs
}

\author{
Matthew L. Wilbur ${ }^{*}$ and W. Keats Wilkie \\ U.S. Army Research Laboratory \\ Vehicle Technology Directorate \\ Hampton, VA 23681
}

\begin{abstract}
The current state-of-the-art in active-twist rotor control is discussed using representative examples from analytical and experimental studies, and the application to rotary-wing UAVs is considered. Topics include vibration and noise reduction, rotor performance improvement, active blade tracking, stability augmentation, and rotor blade de-icing. A review of the current status of piezoelectric fiber composite actuator technology, the class of piezoelectric actuators implemented in active-twist rotor systems, is included.
\end{abstract}

\section{INTRODUCTION}

Rotary-wing flight vehicles, regardless of size or mission, suffer from a number of problems associated with the "edgewise" flight of the rotor system. These problems include high vibratory loads, noise generation, poor performance, instabilities, difficulty maintaining rotor blade track, and limitations on load capacity and forward flight speed. The primary source of these problems is the highly variable aerodynamic environment that the rotor system encounters as it rotates and advances edgewise through the air (see figure 1). Such environments produce periodic vibratory loads that are transmitted directly down the rotor shaft into the fuselage of the vehicle, produce the characteristic "wop-wop" sound associated with rotary-wing vehicles, and accentuate blade-to-blade dissimilarities causing additional vibration, performance, stability, and rotor blade tracking problems.

The rotorcraft research community has studied passive methods to alleviate such concerns for many years; however, acceptable solutions to minimize each of these problems have yet to be established. Nonetheless, advances have been made and rotorcraft systems have reached a stable, albeit generally unacceptable, level of design robustness. Still, work remains to be done and the use of active-control systems is considered by many to be the best method for attacking the many multidisciplinary problems evident in all rotorcraft. ${ }^{1,2}$

The vast majority of the rotorcraft active control research efforts have attempted to eliminate these problems by attacking the source - the aerodynamic loads. Thus, the approach taken has been to modify the periodic aerodynamic loads such that they no longer produce the detrimental effects. The most straightforward method is to modify the rotor blade pitch at harmonic frequencies above the rotor rotational frequency.

Higher Harmonic Control (HHC, see figure 2a), a concept introduced in the 1970 s and ultimately flighttested at full scale, has been shown to reduce vibration and noise by implementing active control of the rotor swashplate to change the pitch at the root of the blades. ${ }^{3}$ Another active control method known as Individual Blade Control (IBC, see figure $2 \mathrm{~b}$ ) typically uses hydraulicallyactuated pitch links to achieve active control of each of the blades independently. Although IBC is, in theory, capable of attacking each of the rotor system problems cited above, the difficulty arises in providing the necessary hydraulic power in the rotating system. Thus, many of the active rotor control concepts being studied currently incorporate on-blade controls, typically trailingedge flaps (figure 2c) or strain-induced blade twist (figure $2 \mathrm{~d}$ ), actuated by piezoelectric devices. These studies have often included wind-tunnel tests of model-scale rotor systems to demonstrate the advantages of active rotor control, and active-flap rotor systems have been demon-
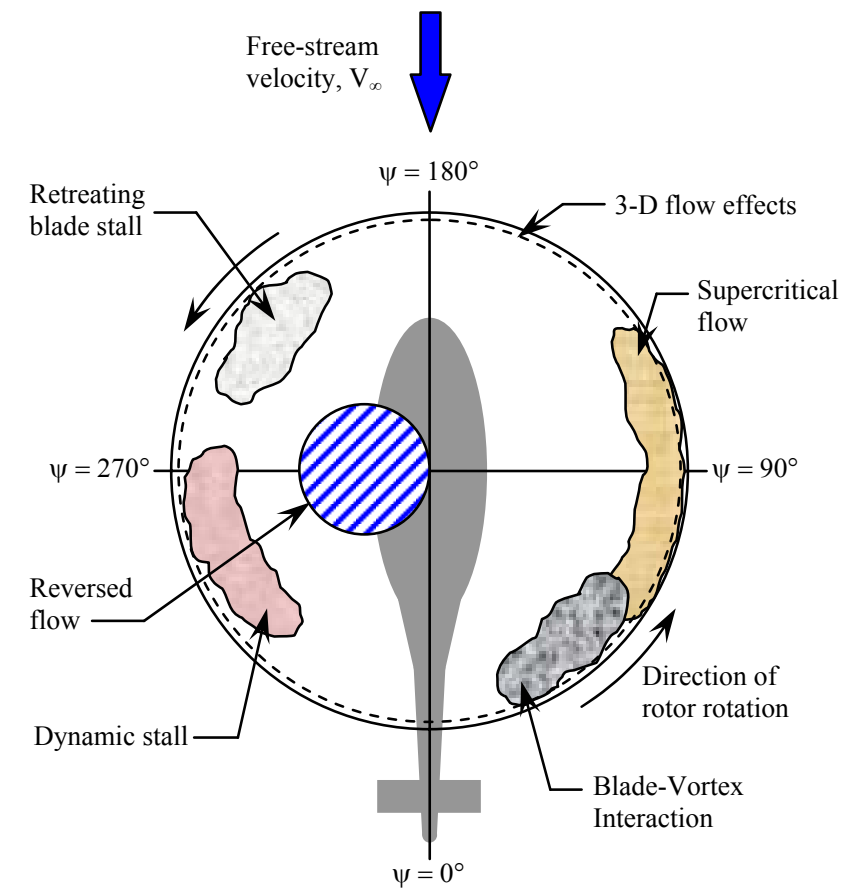

Figure 1. Rotary-wing flight vehicle aerodynamic environment in forward flight. 


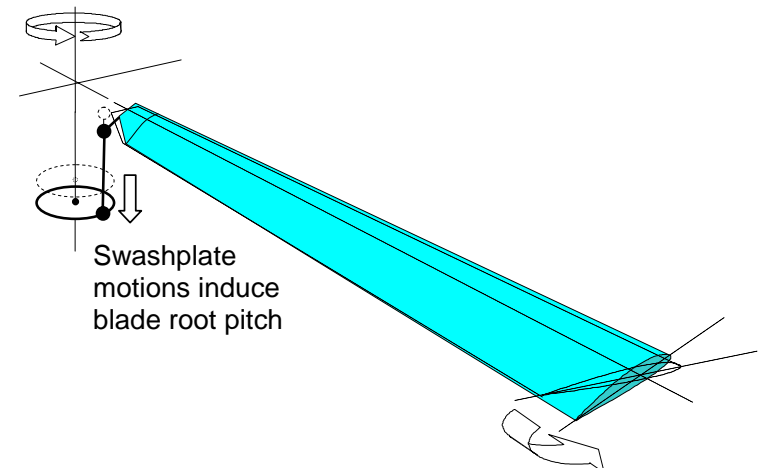

(a) Higher Harmonic Control

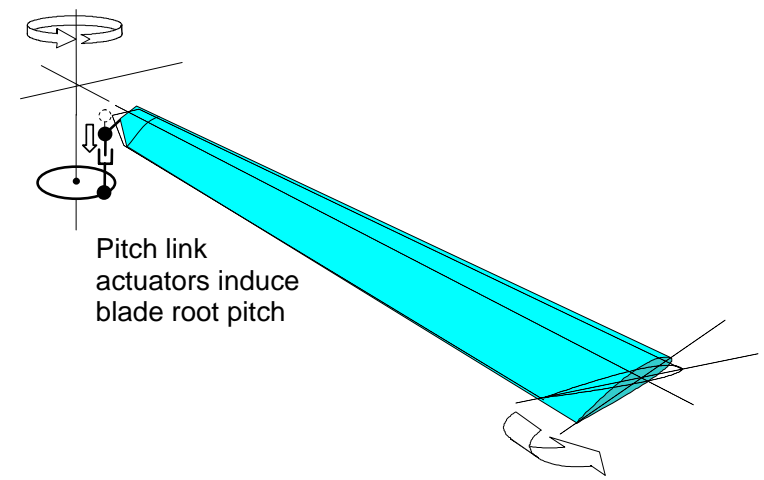

(b) Individual Blade Control

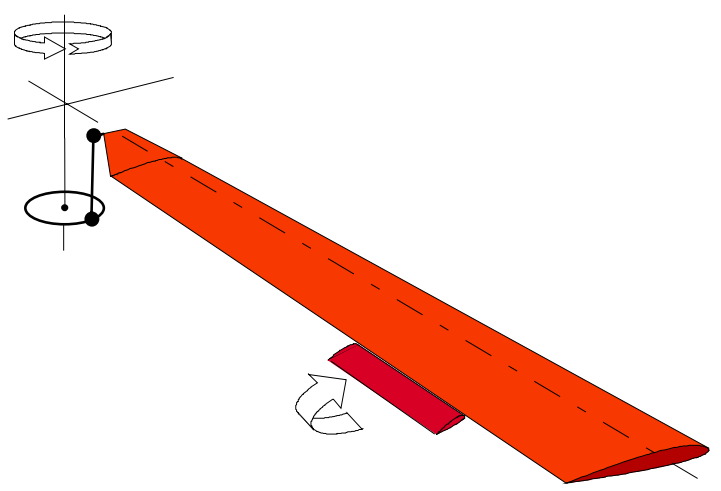

(c) Active trailing-edge flap

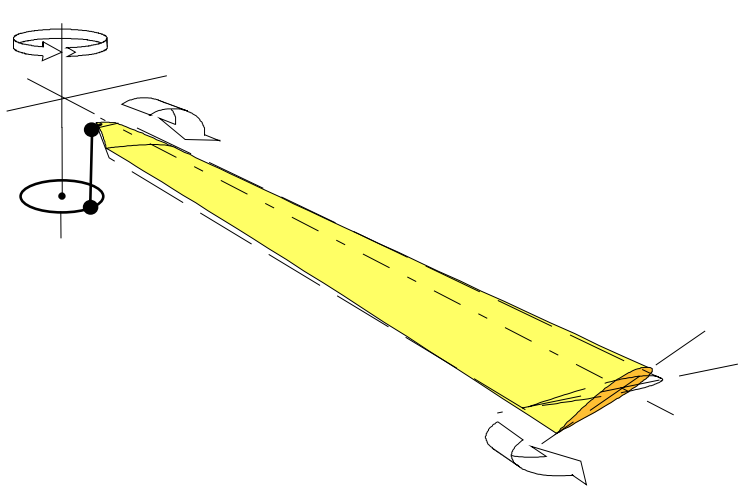

(d) Active-twist rotor

Figure 2. Active rotor control concepts. strated recently in a limited manner at full scale. In general, the results of these studies have been encouraging and have established that on-blade active control is a feasible and efficient approach to attacking typical rotor system problems. Despite this progress, the rotorcraft industry has been reluctant to pursue active rotor control systems aggressively due to the complexity, development costs, and inherent risks of implementing such a system in a man-rated flight vehicle. While it is likely that active rotor control systems will be incorporated eventually in manned rotorcraft, a sensible stepping stone for the successful development of such technologies is the implementation on Unmanned Aerial Vehicles (UAVs) where the risk is reduced.

One active rotor concept that has been examined is the active-twist rotor system. This type of helicopter rotor utilizes piezoelectric fiber composite actuators that are embedded directly within the composite blade structure. These actuators, when excited using electrical voltage, produce strain-induced twisting motions of the blade. One such research effort conducted over the last several years has been the NASA/Army/MIT Active Twist Rotor (ATR) program. The original goal of this program was to study the vibration reduction potential of active-twist rotor systems; however, the study of noise reduction was a secondary goal. A model-scale ATR design was developed for representative Mach- and Froude-scale testing in the heavy gas test medium of the Langley Transonic Dynamics Tunnel (TDT). Forward-flight tests of the ATR were conducted in the TDT in 2000 and in 2002. Results from these tests indicated that significant vibration reduction can be achieved using active-twist control, ${ }^{4-7}$ and that noise reduction comparable to HHC implementations is attainable. ${ }^{8}$ With the completion of this program it has become evident that the applications of active-twist control are far-reaching, and that much of the potential associated with active-twist rotor control has remained untapped. Currently, the use of active-twist control for rotor performance enhancement and active inflight rotor blade tracking are being studied, but other applications such as rotor blade de-icing and stability augmentation are being considered.

The intent of this paper is to introduce the advances in active-twist rotor control technology to the UAV development community. Although the focus of the original ATR research program was directed primarily towards full-scale rotorcraft applications, the technology is equally applicable (and perhaps more so in its current state of development) to systems at UAV scale. UAVs that incorporate a conventional helicopter main-rotor, tailrotor configuration are best suited for direct application of active-twist, including the A-160 Hummingbird, the RQ8A Fire Scout, and the Vigilante; however, the Bell HV911 Eagle Eye, a tiltrotor UAV, is also a candidate for useful active-twist rotor control. This paper will discuss 


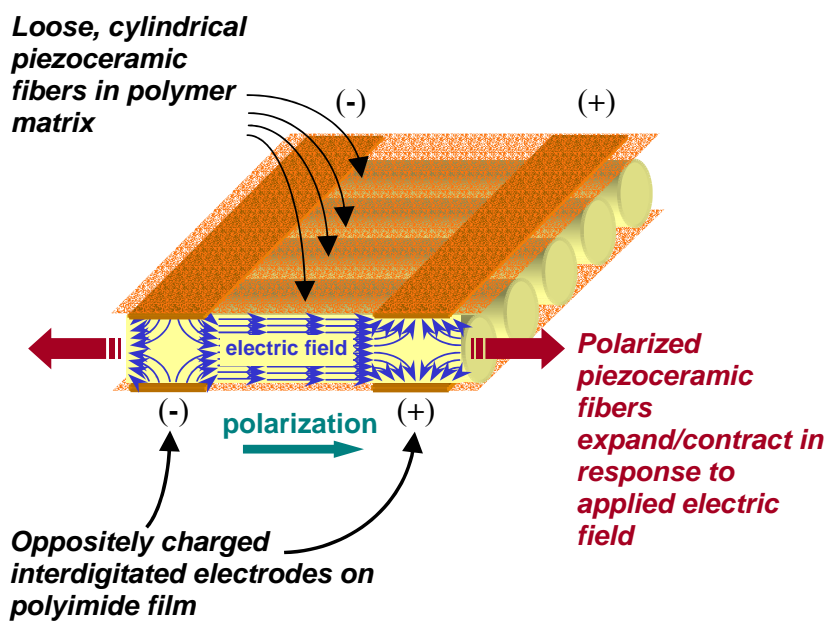

Figure 3. General arrangement of an active fiber composite (AFC) interdigitated electrode piezoelectric fiber composite actuator.

the current state-of-the-art in active-twist rotor control using representative examples from analytical and experimental studies conducted to date. The particular application of active-twist control to rotary-wing UAVs will be considered, with the goal of achieving vibration and noise reduction, rotor performance enhancement, automated blade tracking, and potentially other, as yet unstudied, goals such as rotor blade de-icing and stability augmentation.

\section{PIEZOELECTRIC FIBER COMPOSITE ACTUATOR TECHNOLOGY}

Planar piezoelectric fiber composites are an advanced form of piezoelectric device designed to be embedded within, or bonded to, flexible composite or metallic structures. When subjected to externally applied control voltages, piezoelectric fibers inside the actuator expand or contract, generating actuation forces and deflections in the host structure. These piezoelectrically induced actuation forces may be used dynamically in a feedback arrangement to electronically augment the inherent damping of the combined structure, thereby reducing vibrations or increasing dynamic stability. They may also be used quasistatically, to directly bend or reshape the structure, e.g., to modify its aerodynamic characteristics.

Planar piezocomposite actuators were developed originally to overcome many of the practical difficulties associated with using monolithic piezoceramics for active control of structural composites. ${ }^{9} \quad$ Chief among these difficulties were brittleness of piezoceramic materials, poor conformability (particularly when applied to nonplanar structures), nondirectional nature of strain actuation, and overall low strain energy density. To increase conformability, first generation piezoelectric fiber composite (PFC) actuators were manufactured using

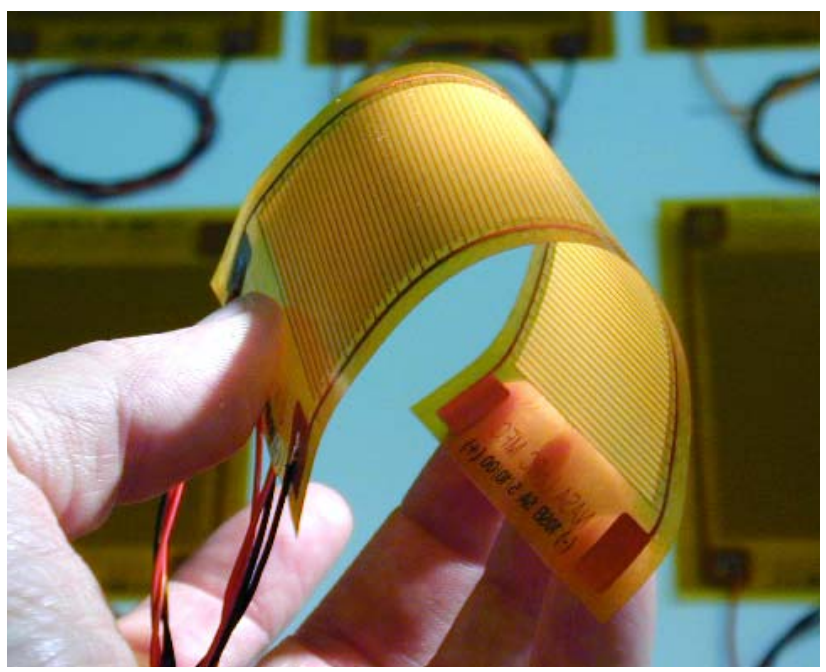

Figure 4. NASA-ARL Macro-Fiber Composite actuator.

a layer of extruded piezoceramic fibers encased in a protective polymer matrix material. Strain energy density was later improved by utilizing interdigitated electrodes to produce electrical fields in the plane of the actuator. The application of in-plane electrical fields permits the piezoceramic elements to produce nearly twice the strain actuation, and four times the strain energy density, of a standard through-plane poled piezoceramic device. The general arrangement of this second generation piezocomposite device, the active fiber composite (AFC) actuator, is shown in figure 3.

Currently, the most sophisticated embodiment of the interdigitated electrode piezocomposite actuator is the NASA-ARL Macro-Fiber Composite (MFC) device. ${ }^{10}$ The MFC (see figure 4) was developed to alleviate many of the manufacturing and performance disadvantages associated with early piezocomposites. ${ }^{11}$ The MFC retains the most advantageous features of the early piezocomposite actuators, namely, high strain energy density, directional actuation, conformability and durability, yet incorporates several new features, chief among these being the use of low-cost fabrication processes that are uniform and repeatable. ${ }^{12,13}$

Early generation piezocomposites utilized individually extruded brittle piezoceramic fibers that were fabricated and handled largely by hand. The MFC uses piezoelectric fiber sheets machined from low-cost piezoceramic wafers using a computer-controlled semiconductor wafer dicing saw. The sheets are easily handled and allow the piezoceramic fibers to be precisely aligned within the actuator package. Producing and handling piezoceramic fibers in precision groups, versus individual pieces, minimizes variations in the active and passive properties of the actuator package. Production costs associated with handling and manufacturing of individual piezoceramic fibers are also reduced. The flat surfaces of the piezoceramic fiber elements also permit a 


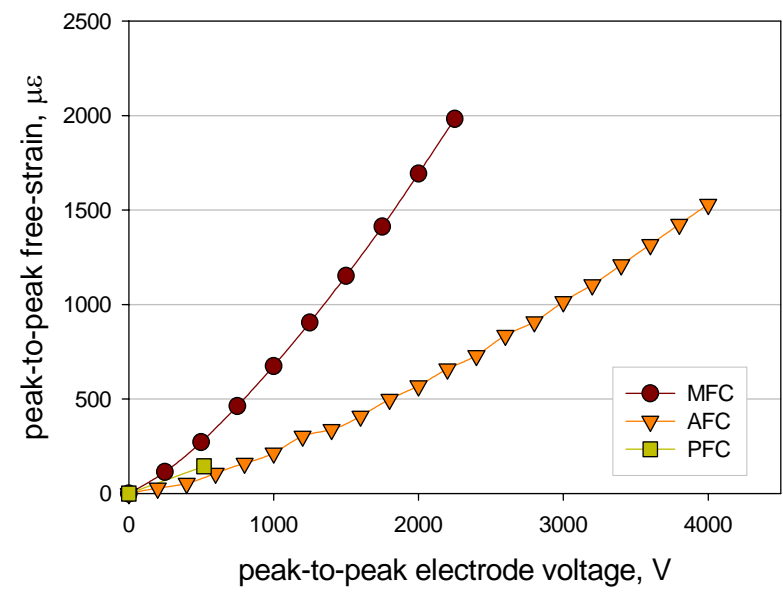

Figure 5. Actuation performance comparison of piezocomposite devices.

greater surface area to be in direct contact with the interdigitated electrodes, which minimizes electric field attenuation by the low dielectric epoxy matrix. As a result, actuation performance (free-strain per volt) of MFC piezocomposites is consistently higher ( $>250 \%$ ) than the earlier round piezoceramic fiber piezocomposites, which often suffer from poor or inconsistent electrode contact as a result of the cylindrical electrode geometry.

Actuation performance of the PFC, the AFC, and the latest version of the MFC are compared in figure 5. ${ }^{14-16}$ The improved electrode interface of the MFC results in its higher free-strain output. The lower voltage requirement of the MFC is due to the smaller relative spacing of the interdigitated electrodes on the MFC as compared to the AFC. The AFC piezocomposite actuators were used successfully with the NASA/Army/MIT Active Twist Rotor Project (ATR), described in the next section. The MFC will be used in an advanced Active Twist Rotor system, being designed currently.

\section{NASA/ARMY/MIT ACTIVE TWIST ROTOR}

The application of piezoelectric fiber composite actuators to control the twist of helicopter rotor blades has been a focus of study since the mid-1990s. Several research efforts have been conducted that have developed model-scale demonstration hardware ${ }^{17-19}$ and one program resulted in the development of a short section of a fullscale active-twist rotor blade. ${ }^{20}$ Each of these programs utilized Active Fiber Composite actuators. The NASA/Army/MIT Active Twist Rotor is the only activetwist system to have been tested in forward-flight operation, and is the source of all results presented herein.

\subsection{Wind Tunnel}

All ATR forward-flight testing was conducted in the Transonic Dynamics Tunnel (TDT, see figure 6), located at the NASA Langley Research Center. The TDT is particularly suited for rotorcraft aeroelastic testing primarily because of three advantages associated with the R-134a (heavy gas) test medium utilized in the tunnel. First, the higher density of R-134a allows model rotor components to be heavier; thereby more easily meeting structural design requirements while maintaining dynamic scaling. Second, the low speed of sound in R-134a (approximately $550 \mathrm{ft} / \mathrm{sec}$ ) permits much lower rotor rotational speeds to match full-scale hover tip Mach numbers and reduces the time-scales associated with active control concepts and dynamic response. Finally, the higher-density environment increases the Reynolds number throughout the test envelope, which models more accurately the full-scale aerodynamic environment of the rotor system.

\subsection{Model Helicopter Rotor Testbed}

ATR testing was conducted on the Aeroelastic Rotor Experimental System (ARES) testbed, shown mounted in the test section of the TDT in figure 7. The ARES is a generic model-scale rotor testbed used for studying aeroelastic phenomena of conventional helicopter rotor systems. Nominal model rotor systems diameters of up to $10 \mathrm{ft}$ may be accommodated on the ARES, which has been specifically developed for testing in the heavy-gas test medium of the TDT.

\subsection{ATR Blades}

The Active Twist Rotor is a 4-bladed articulated rotor system with a rotor radius of 55 inches and blade chord of 4.24 inches. A rectangular blade planform, a NACA 0012 airfoil, and 10 degrees of linear blade twist are used. Each ATR blade utilizes 24 AFC actuators to achieve

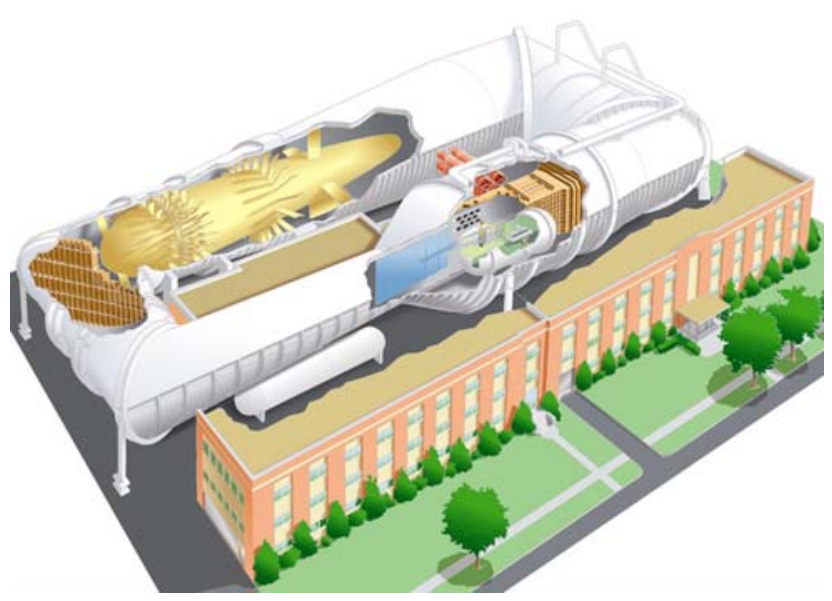

Figure 6. The Langley Transonic Dynamics Tunnel 


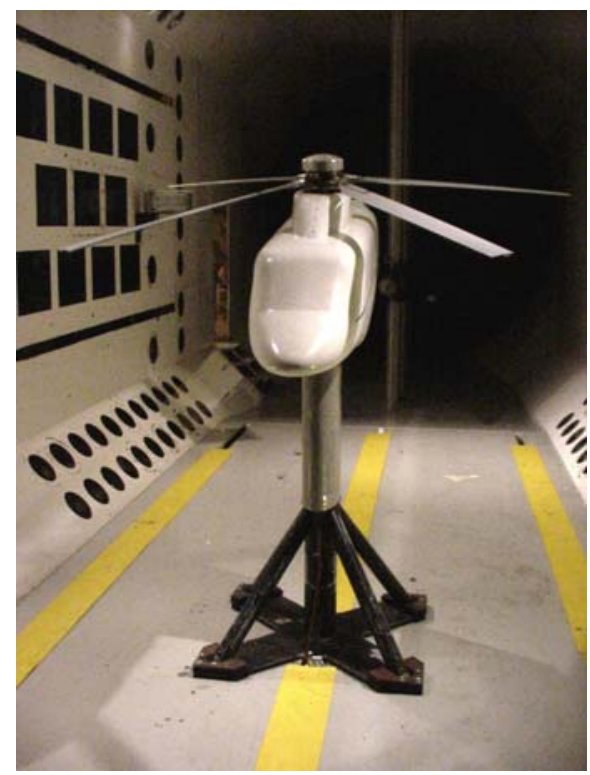

Figure 7. The ARES testbed in the TDT with the ATR hardware installed.

active-twist control. The AFC actuators are embedded directly in the structure of each blade D-spar, as shown in figure 8 , spanning a section of uniform blade structure from $30 \%$ to $98 \%$ of the blade radius ( $0.30 \mathrm{R}$ to $0.98 \mathrm{R}$ ). The AFCs are placed in four layers through the blade thickness and are oriented such that the active strain is applied at $\pm 45^{\circ}$ relative to the blade spanwise axis to generate maximum torsional control of the blades. Four dedicated high-voltage amplifiers, one for each blade, are used to generate high voltage $( \pm 1000 \mathrm{~V})$, low current (60 $\mathrm{mA}$ maximum) power for the independent actuation of each blade.

Blade twist measurements were made during forward-flight testing using a Projection Moiré Interferometry (PMI) optical displacement measurement system. ${ }^{21}$ Most of the data acquired to date has been for actuated frequencies of 3P, 4P, and 5P (three, four, and five times the rotor rotational frequency, $\Omega$ ) because these frequencies have the greatest effect on fixed-system (fuselage) vibratory loads for a four-bladed rotor system like the ATR. Measurements at these frequencies have shown that the ATR is capable of blade twist amplitudes of $1.1^{\circ}$ (at $3 \mathrm{P}$ ) to $1.4^{\circ}$ (at $5 \mathrm{P}$ ) when actuated at voltage amplitudes of $1000 \mathrm{~V}$.

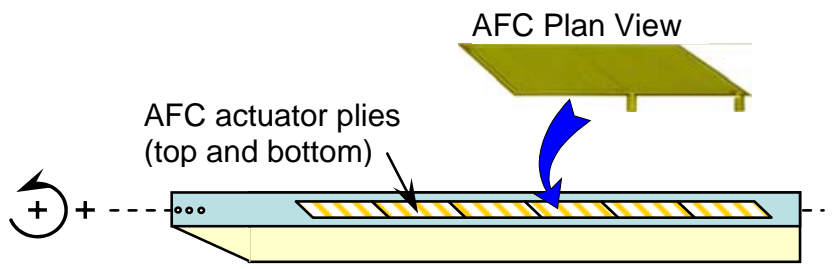

Figure 8. ATR blade planform showing location of AFC actuators. Actuators shown in $-45^{\circ}$ orientation.

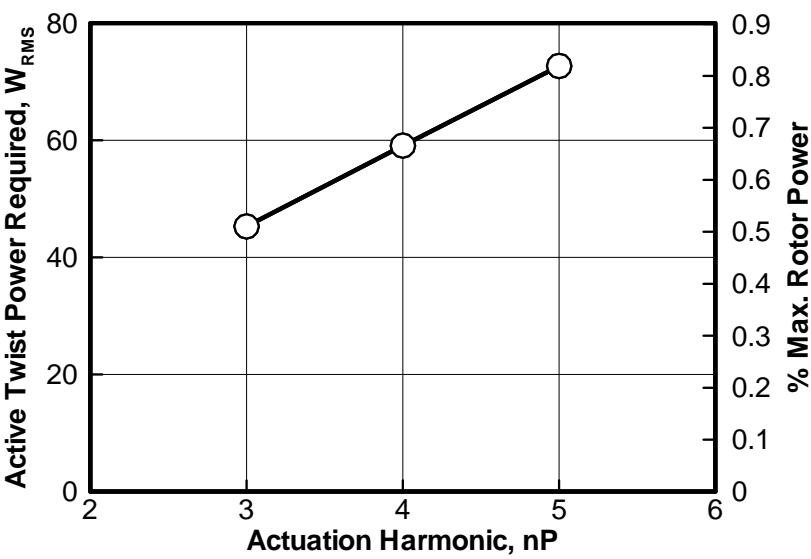

Figure 9. Rotor actuation power required for $1000 \mathrm{~V}$ actuation.

Power requirements for ATR blade actuation are presented in figure 9. The figure presents the power required to actuate all four model-scale ATR blades in $\mathrm{W}_{\text {rms }}$ and as a percentage of rotor power. As shown, the active-twist concept is power efficient, requiring less than $1 \%$ of equivalent rotor power to operate.

\subsection{Vibration Reduction}

The predominant frequency of vibration within the fuselage of a rotary-wing aircraft is at a harmonic frequency equal to the number of blades. For the ATR, this frequency is $4 \mathrm{P}$, or four times the rotor rotational frequency. It can be shown mathematically that the $4 \mathrm{P}$ fixed-system loads are the direct result of $3 \mathrm{P}, 4 \mathrm{P}$, and $5 \mathrm{P}$ loads in the rotating system. Therefore, blade twist actuation at 3P, 4P, and 5P has been used with the ATR to achieve fixed-system vibration reduction. Of these, 3P actuation has been observed to be the most effective in reducing fixed-system vibration. ${ }^{4-7}$

Sample ATR vibration reduction capabilities for a range of forward-flight speed represented by advance ratio, $\mu=V_{\infty} /(\Omega R)$, are presented in figures 10 through 13. The figures present results in a response map format for 3P active-twist actuation at $1000 \mathrm{~V}$ actuation amplitude. Results presented in each figure include the 4P fixed-system longitudinal and vertical shears, and the 4P fixed-system rolling moment. For each plot, solid symbols indicate the unactuated response and the open symbols indicate the response when the ATR blades are actuated at control phases from $0^{\circ}$ to $360^{\circ}$. Radial lines are shown to indicate the response generated when applying $0^{\circ}$ control phase. For all cases, the control phase advances counterclockwise around the plotted data.

The response maps presented in figures 10 through 13 are useful in determining the approximate voltage amplitude and control phase necessary to minimize 4P fixed-system vibratory loads; however, they also provide 


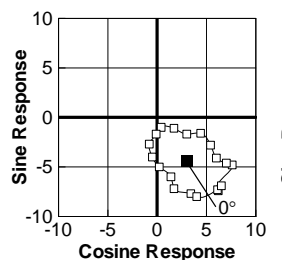

(a) 4P longitudinal shear $\left(F_{x}\right), l b$

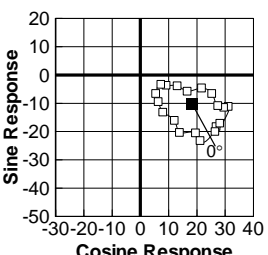

(b) $4 \mathrm{P}$ vertical shear $\left(\mathrm{F}_{\mathrm{z}}\right), \mathrm{lb}$

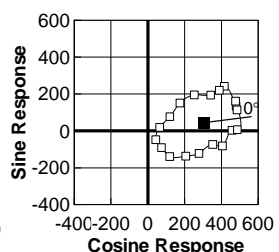

(c) 4P rolling moment $\left(\mathrm{M}_{\mathrm{x}}\right)$, in-lb

Figure 10. Response maps for $\mu=0.140, C_{L}=0.0066, \alpha_{\mathrm{s}}$ $=-1.0^{\circ}$, and $3 \mathrm{P}, 1000 \mathrm{~V}$ actuation.

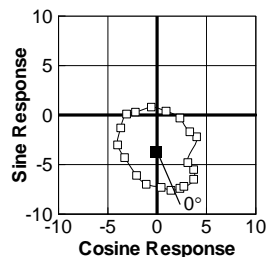

(a) 4P longitudinal shear $\left(\mathrm{F}_{\mathrm{x}}\right), \mathrm{lb}$

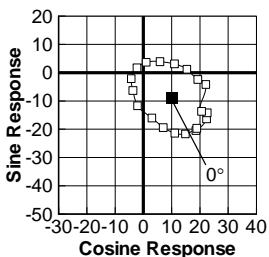

(b) 4P vertical shear $\left(\mathrm{F}_{\mathrm{z}}\right), \mathrm{lb}$

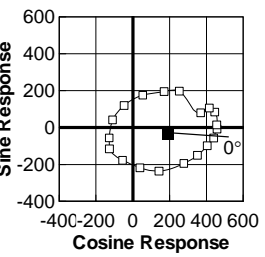

(c) $4 \mathrm{P}$ rolling moment $\left(\mathrm{M}_{\mathrm{x}}\right)$, in-lb

Figure 11. Response maps for $\mu=0.200, C_{L}=0.0066, \alpha_{\mathrm{s}}$ $=-1.0^{\circ}$, and $3 \mathrm{P}, 1000 \mathrm{~V}$ actuation.

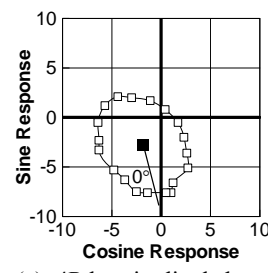

(a) 4P longitudinal shea $\left(\mathrm{F}_{\mathrm{x}}\right), \mathrm{lb}$

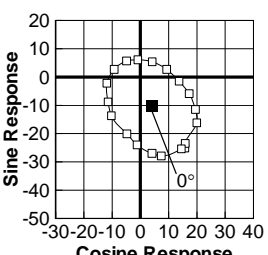

(b) $4 \mathrm{P}$ vertical shear

$\left(\mathrm{F}_{\mathrm{z}}\right), \mathrm{lb}$

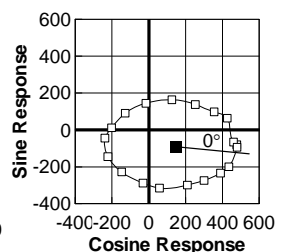

(c) 4 P rolling moment $\left(\mathrm{M}_{\mathrm{x}}\right)$, in-lb

Figure 12. Response maps for $\mu=0.267, C_{L}=0.0066, \alpha_{\mathrm{s}}$ $=-2.0^{\circ}$, and $3 \mathrm{P}, 1000 \mathrm{~V}$ actuation.

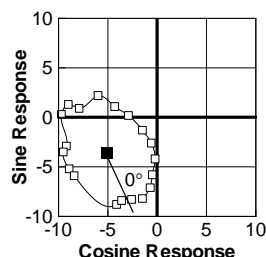

(a) $4 \mathrm{P}$ longitudinal shear $\left(\mathrm{F}_{\mathrm{x}}\right), \mathrm{lb}$

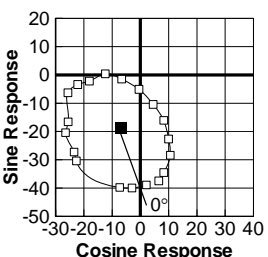

(b) $4 \mathrm{P}$ vertical shear $\left(\mathrm{F}_{\mathrm{z}}\right), \mathrm{lb}$

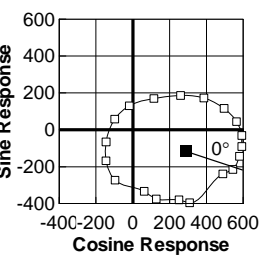

(c) $4 \mathrm{P}$ rolling moment $\left(\mathrm{M}_{\mathrm{x}}\right)$, in-lb

Figure 13. Response maps for $\mu=0.333, C_{L}=0.0066, \alpha_{\mathrm{s}}$ $=-5.0^{\circ}$, and $3 \mathrm{P}, 1000 \mathrm{~V}$ actuation.

a simple format for evaluating the active-twist control authority for vibration reduction. This is assessed qualitatively by examining the size of the "circle" consisting of the actuated response points (open symbols). That is, a larger "circle" corresponds to a greater amount of control authority. Most important, however, is the proximity of the circle to the response map origin because the origin represents the point at which no vibratory load exists. Therefore, response maps in which the circle of actuated response points encloses the origin are examples of conditions where the associated vibratory load may be completely negated using the proper combination of voltage amplitude and control phase. Thus, it is clear that

the three individual fixed-system loads may be eliminated during cruising flight conditions $(\mu=0.200$ and 0.267$)$ and rolling moment may be eliminated at the highest flight speed $(\mu=0.333)$. None of the loads may be eliminated at the lowest flight speed $(\mu=0.14)$, but vibration reductions of $70 \%$ to $90 \%$ are evident. It is important to note that it is not possible to eliminate the individual fixed-system loads simultaneously due to conflicting voltage amplitude and control phase requirements; however, further data analysis has shown that vibration reductions of up to $75 \%$ are achievable when the contribution of each of the $4 \mathrm{P}$ fixed-system loads is weighted equally.

\subsection{Noise Reduction}

The "wop-wop" sound typically associated with helicopters may be traced directly to blade-vortex interaction (BVI) noise and is considered to be the most uncomfortable noise emanating from rotorcraft. BVI noise is particularly prevalent during descending flight conditions where the rotor disk travels through its own wake.

Figures 14 and 15 present sample results of the ATR noise measurements acquired. Figure 14 presents the sound pressure level (SPL) for a frequency band encompassing the BVI noise from 26P through 162P. Figure 15 presents the SPL for a low frequency (LF) band encompassing noise from $2 \mathrm{P}$ to $26 \mathrm{P}$. As shown in the figures, active-twist rotor control was observed to be useful for reducing BVI noise (figure 14), but low frequency noise spectra increased for virtually all actuated flight conditions tested (figure 15). Further details regarding the noise reduction results are available in reference 8 .

\section{UAV APPLICATIONS}

As has been suggested, the applications for activetwist rotor control of UAVs are numerous. Rotary-wing UAVs, like their full-scale counterparts, encounter vibration problems that require some form of compensation, such as the over-design of system components or limiting the desired mission requirements. Active-twist rotor control is a practical alternative to such concessions and can be tailored easily to suit various UAVs, including those that utilize variable rotor speed such as the A-160 Hummingbird.

The applicability of active-twist for noise reduction of UAVs is less clear because of the increase in low frequency spectrum noise and because the greatest BVI noise reduction is attained in descending flight conditions. While the BVI noise reduction is an asset for commercial rotorcraft during approach-to-landing operations over 


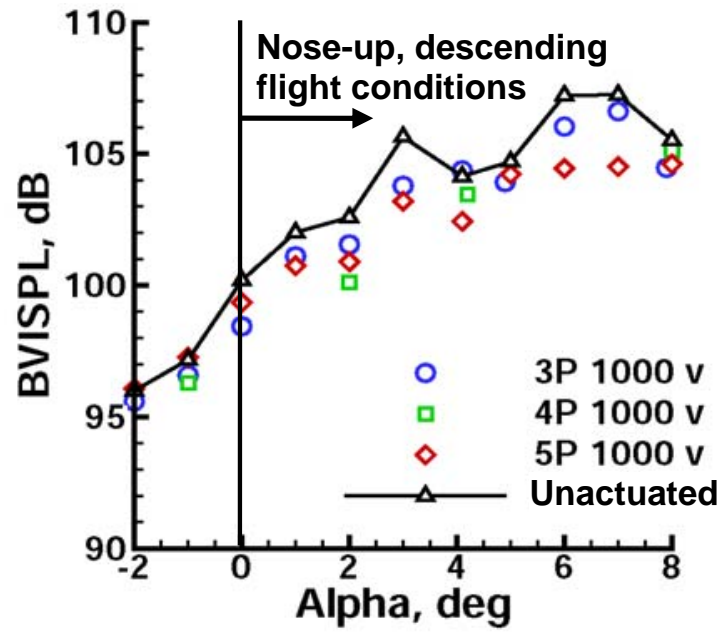

Figure 14. Blade-vortex interaction sound pressure level (BVISPL) noise results for $\mu=0.14$ and $C_{L}=0.0066$.

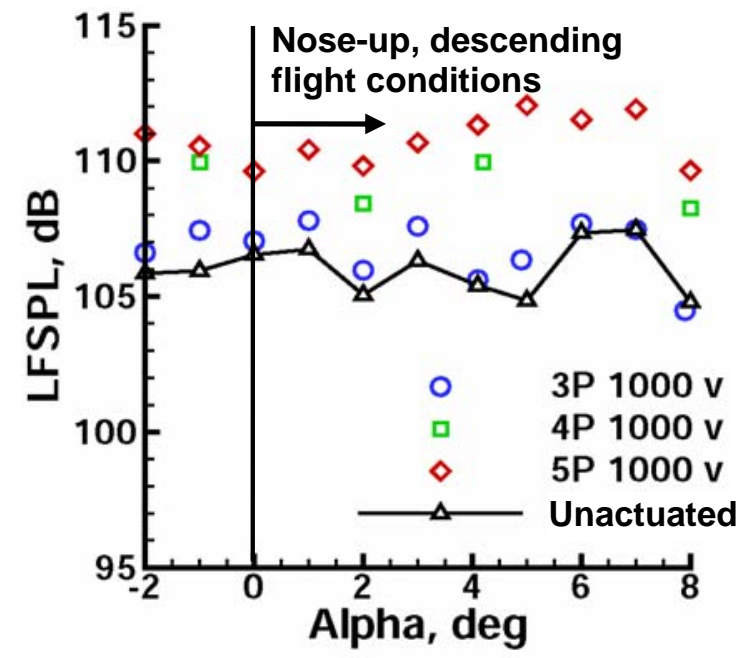

Figure 15. Low frequency sound pressure level (LFSPL) noise results for $\mu=0.14$ and $C_{L}=0.0066$.

populated areas, the applicability to UAVs is not as clear if the primary concern is detectability. Still, noise reduction is one of the available features of active-twist control technology, and may be of some use in the UAV mission.

The other applications for active-twist rotor control have not been as fully researched to date as have been the vibration and noise reduction capabilities. Some data have been acquired, however, suggesting that some rotor performance improvements are available using activetwist control. Limited data acquired during forward-flight testing of the ATR indicated a $1 \%$ improvement in rotor performance at an advance ratio of 0.3; however, unpublished analytical efforts to study performance improvement have suggested that total rotor power reductions of $2 \%$ to $3 \%$ may be possible.
Active-twist rotor control is also capable of real-time rotor blade tracking adjustments to keep each of the rotor blades flying in the plane of the rotor disc. High-speed flight conditions, in particular, often cause individual blades to go out-of-track causing increased vibratory loads and rotor noise, and a reduction in rotor performance. Active rotor blade tracking control, in conjunction with vibration and noise control, could realistically extend the flight envelope of UAVs to encompass greater flight speeds.

Finally, it is feasible that active-twist rotor control could be used to augment rotor stability or be used as a rotor de-icing system. Active swashplate control has been demonstrated previously on a tiltrotor platform as an effective method for improving aeroelastic stability margins. ${ }^{22}$ Similar methods could be utilized with an active-twist rotor system to improve ground- or airresonance and high-speed aeroelastic stability. Also, due to the high operating bandwidth of piezoelectric actuator devices, it is conceivable that an active-twist rotor system could operate as an effective de-icing system. If ice is detected to have accreted on the blade surface, the activetwist frequency could be swept until a resonance is identified in the blade-ice system. By further performing a sine dwell at the resonant frequency, the ice could be made to fracture and subsequently shed from the blade.

\section{CONCLUDING REMARKS}

Active-twist rotor systems for rotary-wing aircraft configurations have received much attention and study over the past decade. The result of these efforts has been to demonstrate the feasibility and potential applications of an active-twist rotor system on model-scale research platforms. While the most significant effort to date has been in the areas of vibration and noise reduction, clear applications for active-twist rotor control in the areas of rotor performance improvement, active blade tracking, and rotor stability improvement are evident. Further applications include the potential for utilizing active-twist as a rotor de-icing system. Further research is required in each of these areas to properly implement active-twist rotor control in a fielded system; however, the technology has approached a level of maturity that should permit the successful application on a UAV.

\section{ACKNOWLEDGEMENTS}

The authors wish to acknowledge the efforts of the many members of the Active Twist Rotor and MFC teams, including those researchers and technicians from NASA, the Army, MIT, the University of Michigan, Sikorsky Aircraft Corporation, and Virginia Tech. 


\section{REFERENCES}

${ }^{1}$ Giurgiutiu, V., “Active-Materials Induced-Strain Actuation for Aeroelastic Vibration Control," Shock and Vibration Digest, Sage Pub., UK, Vol. 32, No. 5, September 2000, pp. 355-368.

${ }^{2}$ Chopra, I., "Status of Application of Smart Structures Technology to Rotorcraft Systems," Journal of the American Helicopter Society, Vol. 45, (4), October 2000, pp. 228-252.

${ }^{3}$ Wood, E. R., Powers, R. W., Hammond, C. E., and Cline, J. H., "On Developing and Flight Testing A Higher Harmonic Control System," Journal of the American Helicopter Society, Vol. 30, (1), January 1985, pp. 3-20.

${ }^{4}$ Wilbur, M. L., Mirick, P. H., Yeager, W. T., Jr., Langston, C. W., Cesnik, C. E. S., and Shin, S. J., "Vibratory Loads Reduction Testing of the NASA/Army/MIT Active Twist Rotor," Journal of the American Helicopter Society, April 2002, pp. 123-133.

${ }^{5}$ Wilbur, M. L., Yeager, W. T., Jr., and Sekula, M. K., "Further Examination of the Vibratory Loads Reduction Results from the NASA/Army/MIT Active Twist Rotor Test." Proceedings of the $58^{\text {th }}$ Annual Forum of the American Helicopter Society, Montreal, Canada, June 11-13, 2002.

${ }^{6}$ Shin, S. J. and Cesnik C. E. S., "Closed-Loop Control Test of the NASA/Army/MIT Active Twist Rotor for Vibration Reduction." Proceedings of the $59^{\text {th }}$ Annual Forum of the American Helicopter Society, Phoenix, AZ May 6-8, 2003.

${ }^{7}$ Bernhard, A. P. F. and Wong, J., "Sikorsky Active Rotor Control Evaluation of the NASA/Army/MIT Active Twist Rotor." Presented at the $59^{\text {th }}$ Annual Forum of the American Helicopter Society, Phoenix, AZ, May 6-8, 2003.

${ }^{8}$ Booth, E. R. and Wilbur, M. L., "Acoustic Aspects of Active-Twist Rotor Control," Journal of the American Helicopter Society, January 2004, pp. 3-10.

${ }^{9}$ Bent, A., "Active Fiber Composite Material Systems for Structural Control Applications," Proceedings, SPIE's 6th International Symposium on Smart Structures and Materials, Newport Beach, CA, March 1-5, 1999.

${ }^{10}$ Wilkie, W., High, J., Mirick, P., Fox, R., Little, B., Bryant, R., Hellbaum, R., and Jalink, A., "Low-Cost Piezocomposite Actuator for Structural Control Applications," Proceedings, SPIE's 7th International Symposium on Smart Structures and Materials, Newport Beach, California, March 5-9, 2000.

${ }^{11}$ Yoshikawa, S., Farrell, M., Warkentin, D., Jacques, R., and Saarmaa, E., "Monolithic Piezoelectric Actuators and Vibration Dampers with Interdigitated Electrodes," Proceedings, SPIE's 6th International Symposium on Smart Structures and Materials, Newport Beach, CA, March 1-5, 1999.
${ }^{12}$ High, J., and Wilkie, W., "Method of Fabricating NASA-Standard Macro-Fiber Piezocomposite Actuators," NASA/TM-2003-212427, ARL TR 2833, June 2003.

${ }^{13}$ Wilkie, W., et al, "Method of Fabricating a Piezoelectric Composite Apparatus," U.S. Patent No. 6,629,341, October 7, 2003.

${ }^{14}$ Williams, R., "Nonlinear Mechanical and Actuation Characterization of Piezoceramic Fiber Composites," Ph.D. dissertation, Virginia Polytechnic and State University, March, 2004.

${ }^{15}$ Wickramasinghe, V., and Hagood, N., "Material Characterization of Active Fiber Composites for Integral Twist-Actuated Rotor Blade Applications," Smart Materials and Structures, Vol. 13, August 2004, pp.1155-1165.

${ }^{16}$ Rodgers,J., and Hagood, N., "Manufacture of Adaptive Composite Plates Incorporating Piezoelectric Fiber Composite Plies," AIAA Paper 95-1096-CP, $36^{\text {th }}$ AIAA/ASME/ASCE/AHS/ASC Structures, Dynamics, and Materials Conference, New Orleans, LA, April 10-13, 1995.

${ }^{17}$ Rodgers, J. P. and Hagood, N. W., "Development of an Integral Twist-Actuated Rotor Blade for Individual Blade Control," Active Materials and Structures Laboratory, AMSL Report \#98-6, Massachusetts Institute of Technology, October 1998.

${ }^{18}$ Cesnik, C. E. S., Shin, S. J., Wilkie, W. K., Wilbur, M. L., and Mirick, P. H., "Modeling, Design, and Testing of the NASA/Army/MIT Active Twist Rotor Prototype Blade," AHS International 55th Annual Forum Proceedings, Montreal, Canada, May 25-27, 1999.

${ }^{19}$ Derham, R., Weems, D., Mathew, M., and Bussom, R., "The Design Evolution of an Active Materials Rotor," American Helicopter Society 57th Annual Forum Proceedings, Washington, DC, May 9-11, 2001.

${ }^{20}$ Weems, D. B, Anderson, D. M., Mathew, M. B., and Bussom, R. C., "A Large-Scale Active-Twist Rotor." Proceedings of the $60^{\text {th }}$ Annual Forum of the American Helicopter Society, Baltimore, MD, June 7$10,2004$.

${ }^{21}$ Fleming, G. A., Soto, H. L., and South, B. W., "Projection Moiré Interferometry for Rotorcraft Applications: Deformation Measurements of Active Twist Rotor Blades," American Helicopter Society 58th Annual Forum Proceedings, Montreal, Canada, June 2002.

${ }^{22}$ Kvaternik, R. G., Piatak, D. J., Nixon, M. W., Langston, C. W., Singleton, J. D., Bennett, R. L., and Brown, R. K., "An Experimental Evaluation of Generalized Predictive Control for Tiltrotor Aeroelastic Stability Augmentation in Airplane Mode of Flight," Journal of the American Helicopter Society, July 2002, pp. 198-208. 